\title{
The phenomenon of biological evolution: 19th century misconception
}

\author{
Dalius Balciunas
}

\begin{abstract}
Scientists still think that biological evolution is driven by the process named natural selection. Perhaps this 19th century notion was indeed a revolutionary idea at the time when it has been introduced. However, now it seems that natural selection hypothesis most probably is wrong. It does not explain, above all, why biological organization arise in the course of evolution. I show, on a rather abstract level of consideration, that exists another explanation why this intriguing phenomenon - life evolution - take place. Here it is argued that biological organization is solely a product of self-replication.
\end{abstract}

Keywords: logistic equation, self-replication, natural selection, competition

\section{Introduction}

Evolutionary biology and ecology are interconnected by a famous equation known as logistic population growth model

$$
\dot{x}=r x\left(1-\frac{x}{K}\right) .
$$

Here $x$ and $\dot{x}$ are the $X$ population density and its net instantaneous growth rate, respectively. $K$ denotes the maximum density that can be sustained under given conditions.

It is amazing that an evolution force of biological world is hidden in this simple mathematical expression. However, logistic equation has two interpretations, depending on how we describe the remaining parameter $r$. The following formula

$$
\dot{x}=r\left[\left(1-\frac{x}{K}\right) x\right]
$$

suggests that $r$ is the instantaneous relative population density growth rate. Then both terms on the right hand side of the equality

$$
\dot{x}=r x-\kappa x^{2}
$$


are real. This means that $r x$ represents real density growth rate at any given moment $t$. Let us give an interpretation of this model. Assume that population produces more individuals than can survive in overcrowded world. In that case individuals inevitably are involved in real negative intraspecific interactions, described by the interference term $-\kappa x^{2}$, which may be named by a common word - competition.

If a number of populations $X_{i}(i=1,2, \ldots, n)$ compete, we write

$$
\dot{x}_{i}=r_{i} x_{i}-\sum_{k} \kappa_{i k} x_{i} x_{k}
$$

Some of them may extinct. A survival criterion for a given population $X_{i}$ is the following inequality

$$
-\sum_{k}\left(\frac{\kappa_{i k} / \kappa_{i i}}{K_{i}}-\frac{1}{K_{k}}\right) x_{k}=\sum_{k}\left(\frac{\kappa_{k k}}{r_{k}}-\frac{\kappa_{i k}}{r_{i}}\right) x_{k} \geq 0 .
$$

From here it follows that populations have more chances to extend their existence if they produce more offspring and compete more successfully with their rivals of other populations.

Such philosophy of life was elaborated by 19th century scientists, most renowned of whom now is A. R. Wallace and C. R. Darwin. They considered interactions between competitors as a driving force of evolution in the natural world of limited resources (Darwin, Wallace, 1858). Known as natural selection, this specifically biological by nature mechanism is generally accepted as an explanation of life evolution. Despite its seemingly biological origin scientists also try to find elements of natural selection process in nonliving world (Eigen, Schuster, 1979; Pross, 2003; Biebricher, Eigen, 2005; Nowak, Ohtsuki, 2008). This is necessary to link prebiological and biological evolution.

\section{Results and hypothesis}

Searching for the physical basis of biological evolution let us look at logistic equation (1) from a different angle

$$
\dot{x}=\left[r\left(1-\frac{x}{K}\right)\right] x .
$$

Here the instantaneous relative population density growth rate is $\left[r\left(1-\frac{x}{K}\right)\right]$. Thus, we conclude from the expression

$$
\dot{x}=r x-\kappa x^{2}
$$


that, as $r x$ is not real instantaneous growth rate, $-\kappa x^{2}$ also does not represent real negative interactions between individuals, but rather the virtual ones. This model describes a formal chemical autocatalytic reaction

$$
\begin{aligned}
& S+X \stackrel{b}{\rightarrow} X+X, \\
& X \stackrel{d}{\rightarrow} S
\end{aligned}
$$

with a constant total mass density $M=s+x$. Really, from the equation for this reaction

$$
\dot{x}=b s x-d x
$$

we deduce

$$
\dot{x}=\varphi x-b x^{2},
$$

if we denote $\varphi \equiv b M-d$. Supposing further that $\varphi>0$ it is easy to write the model in the logistic form (1)

$$
\dot{x}=\varphi x\left(1-\frac{x}{\Phi}\right),
$$

while

$$
\Phi=M-\frac{d}{b} .
$$

Accordingly, adding competitors to this system, we obtain the following set of equations

$$
\dot{x}_{i}=\varphi_{i} x_{i}\left(1-\frac{\sum_{k} x_{k}}{\Phi_{i}}\right) .
$$

As in our previous case competitors will survive only if

$$
-\sum_{k}\left(\frac{1}{\Phi_{i}}-\frac{1}{\Phi_{k}}\right) x_{k}=\sum_{k}\left(\frac{b_{k}}{\varphi_{k}}-\frac{b_{i}}{\varphi_{i}}\right) x_{k} \geq 0 .
$$

This will be the case only if all $\Phi_{i}$ have the same value (Balciunas, 2009) or, what is the same, all $d_{i} / b_{i}(i=1,2, \ldots, n)$ are equal. Therefore, instead of the above expression we write a condition of survival for any $X_{i}$ ( $i=$ $1,2, \ldots, n)$

$$
-\sum_{k}\left(\frac{1}{\Phi_{i}}-\frac{1}{\Phi_{k}}\right) x_{k}=\sum_{k}\left(\frac{b_{k}}{\varphi_{k}}-\frac{b_{i}}{\varphi_{i}}\right) x_{k}=0 .
$$

It is autocatalytic process, or more particularly self-replication, itself that has such property. Indeed for parallel chemical reactions

$$
S \rightleftharpoons X_{i}, \quad i=1,2, \ldots, n,
$$


with forward and backward rate constants $b_{i}$ and $d_{i}$, respectively, we have (for any two "competitors" $X_{j}$ and $X_{k}$ )

$$
\mu_{x_{j}}-\mu_{x_{k}}=A_{k}-A_{j}=\eta\left(\ln \frac{K_{k}}{Q_{k}}-\ln \frac{K_{j}}{Q_{j}}\right)=\eta\left(\ln \frac{K_{k}}{K_{j}}-\ln \frac{x_{k}}{x_{j}}\right)
$$

$\eta$ denotes some coefficient, $\mu_{x_{i}}$ is chemical potential of $X_{i}$ substance, $A_{i}=$ $\mu_{s}-\mu_{x_{i}}, K_{i}=b_{i} / d_{i}, Q_{i}=x_{i} / s, \mu_{x_{j}}-\mu_{x_{k}}$ means virtual "competition" between $X_{j}$ and $X_{k}$. Equilibrium is reached when $\ln \left(x_{k}^{*} / x_{j}^{*}\right)=\ln \left(K_{k} / K_{j}\right)=$ const, where $x_{i}^{*}$ is the equilibrium concentration of $X_{i}$.

Meanwhile for concurrent autocatalytic reactions

$$
\begin{aligned}
& S \stackrel{b_{i} x_{i}}{\rightarrow} X_{i}, \\
& X_{i} \stackrel{d_{i}}{\rightarrow} S, \quad i=1,2, \ldots, n
\end{aligned}
$$

$K_{i}=b_{i} x_{i} / d_{i}$, and we obtain (Balciunas, 2009)

$$
\mu_{x_{j}}-\mu_{x_{k}}=\eta \ln \frac{s_{(j)}^{*}}{s_{(k)}^{*}} .
$$

Here $s_{(i)}^{*}=d_{i} / b_{i}$. An effect of a constant virtual force between competitors $X_{j}$ and $X_{k}$ does not allow coexistence, except in a case when competitors are identical, i.e. when $s_{(j)}^{*}=s_{(k)}^{*}$. The hypothesis presented below is based on this difference between simple virtual "competition" (16) and virtual autocatalytic competition (18).

No matter how self-replicating entities look like, their different populations may coexist permanently if and only if they all are identical. Identity is acquired not only due to individual properties but through various interactions between individuals too. The more complex entities are itself, and the more sophisticated interactions between them occur the more sustainable system can be.

The last statement, I suppose, is a central point of the theory of biological evolution. To support it I will draw a parallel with processes which run against some natural gradient. For them to occur it is necessary that they would be accordingly interconnected with spontaneous processes - such that follow a natural gradient. Coupled chemical reactions is an example (see Caplan, Essig, 1983): portion of free energy, "released" in spontaneous reactions, is "accumulated" in some other chemical processes, which otherwise do not proceed.

In our case we deal with a system that consists of competing self-replicating entities. Here we come across another kind of interlinked processes going in 
opposite directions. We have two processes that run spontaneously. First of all, from a physical point of view, it is unlikely that only one sort of selfreplicators would dominate permanently: new forms of them appear constantly. This process is equivalent to degradation. There are no physical means to stop it. In the second place, differences between self-replicators populations, i.e. between their $\Phi_{i}$ values, create gradients $\Phi_{j}-\Phi_{k}$ which spontaneously tend to eliminate those dissimilarities. As a result of the combination of these two spontaneous processes organized communities of organized self-replicators arise in the course of evolution. Simply because such structures are able to maintain their components identity in average by the virtue of interactions between them and due to the complexity of components itself.

We may formalize this in the following way. Using (13) introduce $\Psi_{i}$ notion by defining

$$
\sum x_{k}=\Psi_{i} \Phi_{i}, \quad i=1,2, \ldots, n
$$

where $\Psi_{i}>0$ is a function showing the state of a population. If $\Psi_{i}>1$ then a respective population is suppressed by its competitors. Rewrite (20) in the form

$$
\sum x_{k}=\Psi \Phi_{i}+\left(\Psi_{i}-\Psi\right) \Phi_{i}, \quad i=1,2, \ldots, n,
$$

here $\Psi=\sum \Psi_{k} x_{k} / \sum x_{k}$ is a specific value of $\Psi_{i}(i=1,2, \ldots, n)$. Expression $\left(\Psi_{i}-\Psi\right)$ is equivalent to (15) sum. Then the following equations reflect the evolution of the system

$$
\begin{aligned}
& \lim _{t \rightarrow \infty}\left(t-t_{0}\right)^{-1} \int_{t_{0}}^{t} \sum x_{k} d \tau=\lim _{t \rightarrow \infty}\left(t-t_{0}\right)^{-1}\left(\int_{t_{0}}^{t} \Psi \Phi_{i} d \tau+\int_{t_{0}}^{t}\left(\Psi_{i}-\Psi\right) \Phi_{i} d \tau\right), \\
& i=1,2, \ldots, n .
\end{aligned}
$$

Because $\lim _{t \rightarrow \infty}\left(t-t_{0}\right)^{-1} \int_{t_{0}}^{t} \sum x_{k} d \tau=\Phi^{*}$, where $\Phi^{*}$ is some equilibrium value, we obtain from $(22)$

$$
\begin{aligned}
& \Phi^{*}=\lim _{t \rightarrow \infty}\left(t-t_{0}\right)^{-1} \int_{t_{0}}^{t} \Psi \Phi_{i} d \tau+\lim _{t \rightarrow \infty}\left(t-t_{0}\right)^{-1} \int_{t_{0}}^{t}\left(\Psi_{i}-\Psi\right) \Phi_{i} d \tau, \\
& i=1,2, \ldots, n .
\end{aligned}
$$

The second limit should converge to zero. Only in this case mean $\Phi_{i}(i=$ $1,2, \ldots, n)$ values are equal to $\Phi^{*}$. That allows competitors to survive.

Hypothesis presented here resembles another explanation of biological evolution and diversity. Once again imagine two spontaneous processes. One of them creates biodiversity at a rate $v^{+}$, the other reduces it at a rate $v^{-}$. 
What we obtain depends on the rates of these processes. The system may even come to a stationary point if the rates become equal. Such system is indifferent regarding of what species it consists. This scheme describes so called neutral models of evolution and biological diversity (Kimura, 1982, 1989; Hubbell, 2001). Our model differs from these ones in that that the process which diminish biodiversity has a peculiar property. It requires that only identical variants must remain, no matter how many of them will survive. This difference is essential to the final result. Strictly neutral theories of evolution and diversity are the extreme cases of our model. They would be valid if all self-replicating forms are identical by identity - the situation in general very improbable. Real communities of biological species hardly are "neutral" accumulation of various forms; they rather represent strongly organized systems, which elements have attained relative identity during billions of years of interrelations. Briefly, virtual competition between self-replicators creates organization. No intelligence is required a priori to initiate and drive biological evolution. Evolution is only one more manifestation of self-organization in despite of its seemingly unnatural character.

Natural selection theory does not make clear what drove chemical evolution in prebiological world, when selection process has begun and how it led to the complexity that is characteristic feature of biological systems. Meanwhile, scientists up to now are sure that natural selection mechanism is a physical reality responsible for the diversity of living beings. For example, trying to explain prebiological evolution in the terms of natural selection Nowak and Ohtsuki (2008) proclaim that selection preceded self-replication. Such standpoint shows that scientists do not comprehend the special dynamics of concurrent autocatalysis. I think that natural selection theory is a 19th century relic: darwinian evolutionary force appears to be something more subtle. Darwin indeed has made a great proposal (Darwin, 1859). Namely, species interactions or, in his own words, "the struggle for existence" are responsible for their evolution. However, what Darwin saw as an evolutionary force is merely a result of evolution. All sophisticated forms of species and all fanciful ways of their interactions with living and physical environment have developed in response to make species identical. One and a half century ago physical forces of life evolution were hidden from the scientific community of those days. Now it appears that autocatalytic mechanism may be responsible, at least partly, for darwinian evolution, which progressively has led to the formation of organized biosphere. 


\section{References}

Balciunas, D. 2009. The driving force of life evolution. Neuroquantology, 7: $176-180$.

Biebricher, C.K., M. Eigen. 2005. The error threshold. Virus Res., 107: 117-127.

Caplan, S.R., A. Essig. 1983. Bioenergetics and linear nonequilibrium thermodynamics. The steady state. Harvard University Press, Cambridge.

Darwin, C.R. 1859. On the origin of species by means of natural selection, or the preservation of favoured races in the struggle for life. John Murray, London; (http://darwin-online.org.uk).

Darwin, C.R., A.R. Wallace. 1858. On the tendency of species to form varieties; and on the perpetuation of varieties and species by natural means of selection. Jl Proc. Linn. Soc. Lond. Zool., 3: 46-50; (http://darwinonline.org.uk).

Eigen, M., P. Schuster. 1979. The hypercycle. A principle of natural self-organization. Springer-Verlag, Berlin.

Hubbell, S.P. 2001. The unified neutral theory of biodiversity and biogeography. Princeton University Press, Princeton, New Jersey.

Kimura, M. The neutral theory as a basis for understanding the mechanism of evolution and variation at the molecular level. 1982. In Molecular evolution, protein polymorphism and the neutral theory, ed. M. Kimura. Springer-Verlag, Berlin: 3-56.

Kimura, M. 1989. The neutral theory of molecular evolution and the world view of the neutralists. Genome, 31: 24-31.

Nowak, M.A., H. Ohtsuki. 2008. Prevolutionary dynamics and the origin of evolution. Proc. Natl Acad. Sci. USA., 105: 14924-14927.

Pross, A. 2003. The driving force for life's emergence: kinetic and thermodynamic considerations. J. theor. Biol., 220: 393-406. 